\title{
Temporal fine structure of all-normal dispersion fiber supercontinuum
}

\author{
Anupamaa Rampur ${ }^{1}$, Dirk-Mathys Spangenberg ${ }^{1}$, Grzegorz Stępniewski²,3, Dominik Dobrakowski², Karol \\ Tarnowski $^{4}$, Karolina Stefańska ${ }^{4}$, Adam Paździor ${ }^{5}$, Pawel Mergo ${ }^{5}$, Tadeusz Martynkien ${ }^{4}$, Thomas \\ Feurer $^{1}$, Mariusz Klimczak ${ }^{2}$, Alexander M. Heidt ${ }^{1}$ \\ 1. Institute of Applied Physics, University of Bern, Sidlerstrasse 5, 3012 Bern, Switzerland \\ 2. Faculty of Physics, University of Warsaw, Pasteura 5, 02-093 Warsaw, Poland \\ 3. Lukasiewicz Research Network - Institute of Microelectronics and Photonics, Al. Lotników 32/46, 02-668 Warsaw, Poland \\ 4. Department of Optics and Photonics, Wroclaw University of Science and Technology, Wybrzeze Wyspianskiego 27, 50-370 Wroclaw, \\ Poland \\ 5. Laboratory of Optical Fiber Technology, Maria Curie-Sklodowska University, pl. M. Curie-Sklodowskiej 3, 20-031 Lublin, Poland
}

Many ultrafast optics applications require uniform and smooth spectral and temporal intensity profiles of ultrashort laser pulses. All normal dispersion (ANDi), coherent light pulses from fiber-based supercontinuum (SC) are often assumed to fulfill these requirements, backed up by many numerical simulations. However, detailed experimental data on the spectro-temporal characteristics of ANDi SC pulses is scarce.

We will discuss detailed results on experimental spectro-temporal characterization of coherent SC pulses generated in several implementations of ANDi photonic crystal fibers (PCF) pumped by a commercial ultrafast Er:fiber laser. The PCFs were drawn using either highly nonlinear silicate soft glasses or germanium-doped and pure silica glass, and their dispersion was engineered to realize broadband ANDi profiles optimized for the pump wavelength centred around $1560 \mathrm{~nm}$. Both polarization-maintaining (PM) and non-PM fibers were realized and investigated. Full-octave spanning group delay profiles were recorded and analyzed using the time-domain ptychography (TDP) [1].

(a) Measured

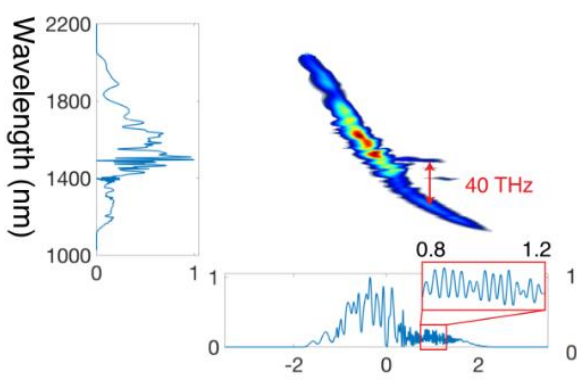

(b) Simulated - full

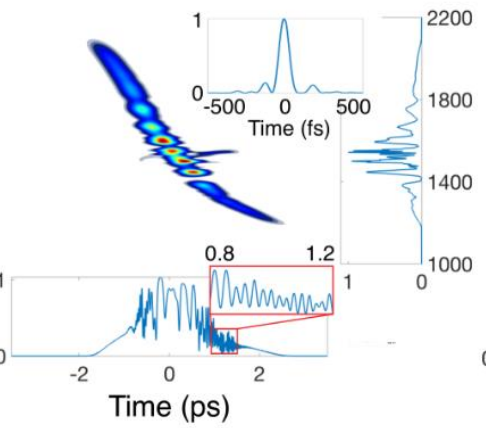

(c) Simulated - filtered

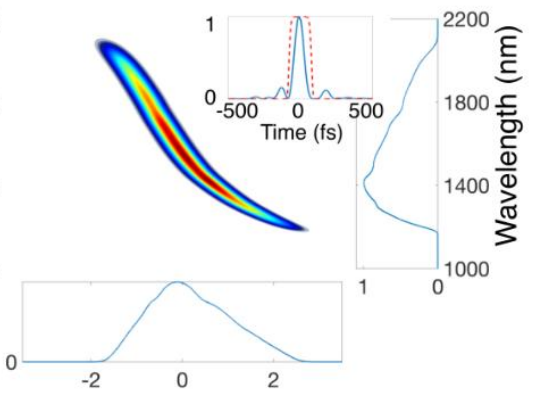

Fig. 1 Projected axes spectrograms of SC pulses generated in a PM ANDi fiber. (a) Measured; (b) simulated considering full amplitude and phase of measured pump pulse; (c) simulated considering temporally filtered pump pulse without side-peaks or pedestal. The projected axes show the retrieved or simulated spectral and temporal profiles, respectively, both in linear scale. Top insets in (b) and (c) show the pump pulse shape taken in the respective simulations.

Our measurements reveal that the SC pulses emitted from all the investigated ANDi fibers exhibit intricate pulse shapes, a considerable amount of temporal fine structure, and fast oscillations on the scale of tens of femtoseconds. This is in contrast to the smoothness and simplicity of the temporal envelopes typically obtained in numerical simulations and observed in previous experiments using fundamental solitons as pump sources [2]. We trace the origin of these observed complex features to imperfections of the pump pulse, such as side pulses and low-level pedestals, which are common in high energy femtosecond Er:fiber-based systems [3]. We also observed spectro-temporal structures of SC pulses generated in weakly birefringent fiber samples that are consistent with signatures of incoherent noise amplification due to polarization modulation instability (PMI) and the nonlinear coupling of parametric four-wave mixing (FWM) and stimulated Raman scattering (SRS) discussed in numerical studies previously [4]. Our results highlight the importance of the pump source and highly birefringent, PM fibers for high-quality SC generation and provide important guidance for the design and application of ANDi SC sources in ultrafast photonics.

\section{Example References}

[1] D. Spangenberg, P. Neethling, E. Rohwer, M. H. Brügmann, and T. Feurer, "Time-domain ptychography," Phys. Rev. A 91, 21803 (2015).

[2] A. Okamura, Y. Sakakibara, E. Omoda, H. Kataura, and N. Nishizawa, "Experimental analysis of coherent supercontinuum generation and ultrashort pulse generation using cross-correlation frequency resolved optical gating (X-FROG)," JOSA B 32, 400-406 (2015).

[3] D. Brida, G. Krauss, A. Sell, and A. Leitenstorfer, "Ultrabroadband Er: fiber lasers," Laser \& Photonics Reviews 8, 409-428 (2014),

[4] I. B. Gonzalo, R. D. Engelsholm, M. P. Sørensen, and O. Bang, "Polarization noise places severe constraints on coherence of all-normal dispersion femtosecond supercontinuum generation," Scientific Reports 8, 1-13 (2018). 